\title{
Effects of Neuronic Shutter Observed in the EEG Alpha Rhythm
}

\author{
(D)Kevin E. Alexander, ${ }^{1,4}$ - Justin R. Estepp, ${ }^{1,2}$ and SSherif M. Elbasiouny ${ }^{1,3}$
}

\author{
https://doi.org/10.1523/ENEURO.0171-20.2020
}

${ }^{1}$ Department of Biomedical, Industrial, and Human Factors Engineering, College of Engineering and Computer Science, Wright State University, Dayton, $\mathrm{OH} 45435,{ }^{2} 711$ th Human Performance Wing, Air Force Research Laboratory, Wright-Patterson AFB, OH 45433, ${ }^{3}$ Department of Neuroscience, Cell Biology, and Physiology, Boonshoft School of Medicine and College of Science and Mathematics, Wright State University, Dayton, $\mathrm{OH} 45435$, and ${ }^{4} \mathrm{Oak}$ Ridge Institute for Science and Education, Air Force Research Laboratory, Wright-Patterson AFB, OH 45433

\begin{abstract}
The posterior alpha $(\alpha)$ rhythm, seen in human electroencephalogram (EEG), is posited to originate from cycling inhibitory/excitatory states of visual relay cells in the thalamus. These cycling states are thought to lead to oscillating visual sensitivity levels termed the "neuronic shutter effect." If true, perceptual performance should be predictable by observed $\alpha$ phase (of cycling inhibitory/excitatory states) relative to the timeline of afferentiation onto the visual cortex. Here, we tested this hypothesis by presenting contrast changes at near perceptual threshold intensity through closed eyelids to 20 participants (balanced for gender) during times of spontaneous $\alpha$ oscillations. To more accurately and rigorously test the shutter hypothesis than ever before, $\alpha$ rhythm phase and amplitude were calculated relative to each individual's retina-to-primary visual cortex (V1) conduction delay, estimated from the individual's C1 visual-evoked potential (VEP) latency. Our results show that stimulus observation rates (ORs) are greater at a trough than a peak of the posterior $\alpha$ rhythm when phase is measured at the individual's conduction delay relative to stimulus onset. Specifically, the optimal phase for stimulus observation was found to be $272.41^{\circ}$, where ORs are $20.96 \%$ greater than the opposing phase of $92.41^{\circ}$. The perception-phase relationship is modulated by $\alpha$ rhythm amplitude and is not observed at lower amplitude oscillations. Collectively, these results provide support to the "neuronic shutter" hypothesis and demonstrate a phase and timing relationship consistent with the theory that cycling excitability in the thalamic relay cells underly posterior $\alpha$ oscillations.
\end{abstract}

Key words: alpha rhythm; EEG; visual-evoked potential; neuronic shutter; visual conduction delay

\section{Significance Statement}

After accounting for neural conduction delays, we found that threshold intensity stimuli are observed at higher rates when the $\alpha$ wave is at a trough phase than at a peak phase, but only when $\alpha$ amplitude is high. Our results were derived using methods consistent with a specific hypothesis about a mechanism of visual perception, considering the structure, physiology, and transmission delays in the visual system. The results of this rigorous study design add support to the neuronic shutter hypothesis and are consistent with the theory that posterior $\alpha$ reflects cycling excitability in thalamic relay cells, thereby gating the flow of visual information.

\section{Introduction}

The posterior alpha $(\alpha)$ rhythm, a $7-$ to $13-\mathrm{Hz}$ oscillation, is a notable characteristic of the human electroencephalogram

Received April 30, 2020; accepted September 10, 2020; First published September 23, 2020.

The authors declare no competing financial interests.
(EEG) over the occipital cortex, especially when eyes are closed (Berry and Wagner, 2015). This is thought to assist attention regulation, as its characteristics are often found to

Author contributions: K.E.A., J.R.E., and S.M.E. designed research; K.E.A. performed research; K.E.A. analyzed data; K.E.A., J.R.E., and S.M.E. wrote the paper. 
correlate with attention over specific sensory cortices (Sauseng et al., 2005; Ben-Simon et al., 2013), possibly a gating mechanism to limit the flow of sensory information (Cooper et al., 2003; Janssens et al., 2018). This rhythm has analogs in the sensorimotor (Pineda, 2005; Bazanova and Vernon, 2014) and auditory cortices (Tiihonen et al., 1991; Weisz et al., 2011), with both appearing to provide similar gating. This regulatory function is further demonstrated by the observation that $\alpha$ activity increases during introspective behavior (Ancoli and Green, 1977) and behaviors involving internally directed attention such as imagination (Cooper et al., 2003), mental arithmetic (Ray and Cole, 1985), autobiographical recall (Yue et al., 2013), and meditative practices (Tenke et al., 2017), presumably because external sensory information is distractive during these behaviors.

However, the cellular mechanism and functional significance of $\alpha$ oscillations remains unclear. In the 1950s, $\alpha$ oscillations were proposed to represent a "neuronic shutter" (Callaway and Alexander, 1960) to down-sample incoming sensory information to reduce processing load, and similar discrete perceptual processes are proposed today (VanRullen and Koch, 2003; VanRullen et al., 2014). This shutter is thought to occur at the lateral geniculate nucleus (LGN), which relays visual information to primary visual cortex (V1). At times, LGN relay cells burst-fire at $10 \mathrm{~Hz}$, with a hyperpolarized refractory period between bursts (Lopes da Silva, 1991; Sherman, 2001; Timofeev and Bazhenov, 2005; Alexander et al., 2006; Timofeev and Chauvette, 2011). If many of these cells fire synchronously, visual afferentation during a widespread refractory period is less likely to be relayed to V1, resulting in a $10-\mathrm{Hz}$ visual shutter. Each burst in LGN relay cells results in a large EPSP at V1, measured as a negativity in the occipital EEG (Timofeev and Bazhenov, 2005), with higher amplitudes indicating more synchronous excitation (Pfurtscheller et al., 1996), resulting, as hypothesized by this model, from LGN excitatory volleys. Thus, the $\alpha$ rhythm is thought to reflect cycling excitability in the LGN visual-relay cells, which would result in cycling visual sensitivity in phase with the $\alpha$ oscillation. Evidence of this mechanism producing $\alpha$ oscillations has been found in animal models with the associated phasic gating of sensory transfer (Lorincz et al., 2009; Chen et al., 2016). The hypothesis of the present study was based on this model; however, we did not directly test the underlying cellular mechanism.

To more accurately measure the $\alpha$ phase relationship to perceptual performance, we accounted for neural

S.M.E. was supported by Ball Aerospace under contract to the United States Air Force, the United States National Institute of Health-National Institute of Neurological Disorders and Stroke and National Institute on Aging Grant NS091836, and the National Academy of Sciences (NAS) and the United States Agency for International Development, NAS Subaward 2000009148. Any opinions, findings, conclusions, or recommendations expressed in this article are those of the authors alone, and do not necessarily reflect the views of USAID or NAS.

Correspondence should be addressed to Sherif M. Elbasiouny at sherif. elbasiouny@wright.edu.

https://doi.org/10.1523/ENEURO.0171-20.2020

Copyright @ 2020 Alexander et al.

This is an open-access article distributed under the terms of the Creative Commons Attribution 4.0 International license, which permits unrestricted use, distribution and reproduction in any medium provided that the original work is properly attributed. conduction delays: if a stimulus strikes the retina at $t 0$, that information arrives at the LGN at a later time, $t 1$. Therefore, the 11 LGN excitatory state determines whether the information is relayed to V1. Further, the LGN excitatory state is measurable even later in the EEG, as V1 EPSPs at $t 2$. Thus, to to $t 2$ represents the retina-to-V1 conduction delay: At $t 2$, we can assess the $t 1$ LGN excitability, the time of afferentation of the to stimulus. This is the first study, to our knowledge, to directly examine the relationship between visual observation and spontaneous $\alpha$ phase relative to each individual's conduction delay, which advances the accuracy and rigor for testing the shutter hypothesis.

This study's objective is to more robustly test the hypothesis that posterior $\alpha$ reflects cyclic excitability of the visual system with a phase and timing relationship predicted by underlying physiological model and conduction delays. Therefore, we presented visual stimuli (to) to participants during spontaneous occipital $\alpha$ activity, then measured $\alpha$ amplitude and phase at $t 2$ (estimated by the individual's C1 visual-evoked potential (VEP) component peak latency; Di Russo et al., 2002). We predicted that visual stimuli at to would be more likely observed with $\alpha$ phase at a trough at $t 2$ (assumed to indicate $t 1$ LGN excitation) than at a peak ( $t 1$ LGN inhibition). Additionally, this effect was predicted to be greater at high $\alpha$ amplitudes, indicating greater cellular excitatory state synchrony. We found that participants responded to $20.96 \%$ more stimuli with $\alpha$ phase at a trough versus a peak at $t 2$ when $\alpha$ amplitude was high. At low amplitude, no significant phase effect on observation rate (OR) was observed. These results support our hypothesis that the $\alpha$ rhythm reflects cyclic excitatory states in the visual system resulting in a visual shutter effect.

\section{Materials and Methods}

\section{Participants}

$\alpha$ Activity varies largely both within (Gonçalves et al., 2006) and between (Wieneke et al., 1980) individuals. For this study, we needed to ensure stimuli were able to be presented during times of observable (e.g., stationary) $\alpha$ oscillations and therefore selected participants who more readily and reliably generated observable occipital $\alpha$ activity. This selection was made by observing EEG activity as the participants practiced the visual sensitivity task with eyes closed during an earlier portion of the experimental session. Participants who did not readily and reliably produce occipital $\alpha$ oscillations during this practice period were excluded from further participation.

In total, 41 participants were recruited for this study. Based on the observable $\alpha$ criteria described above, 21 participants were excluded from further participation. The remaining 20 healthy participants (10 males/10 females, mean age: 22.3 years, range: $18-28$ years) completed two tasks: one to estimate their individual conduction delay; the other to investigate their visual sensitivity at different phases of $\alpha$ oscillations. The experimental protocol was approved by the Institutional Review Boards of Wright 
A

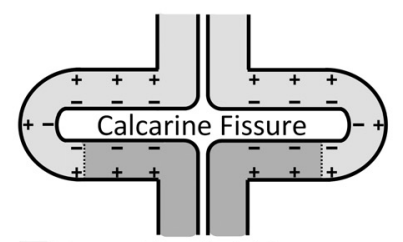

Upper Visual Field

Lower Visual Field
B

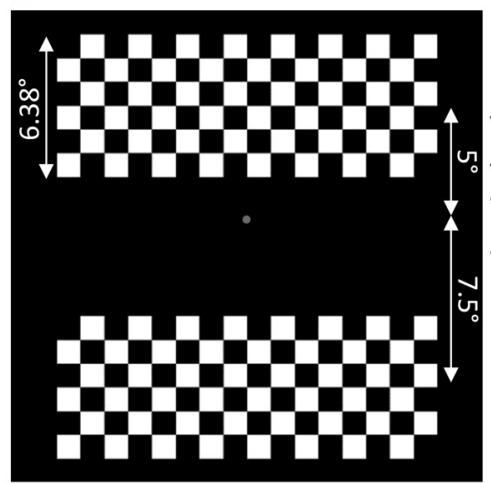

C

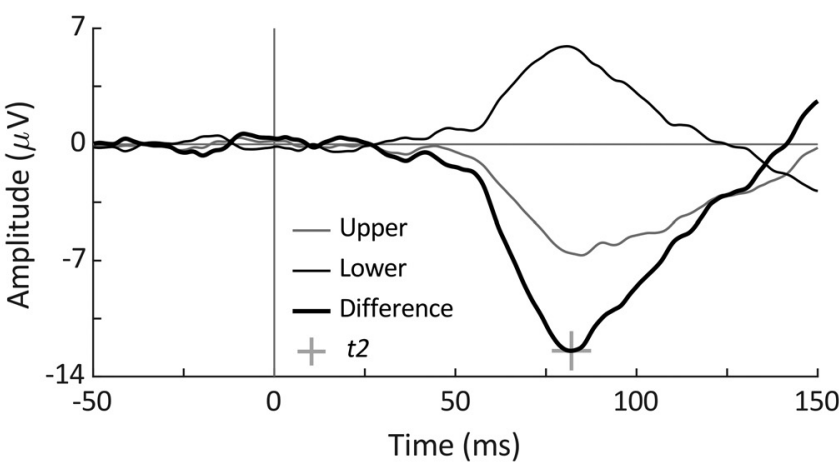

Figure 1. $\boldsymbol{A}$, According to the cruciform model of the calcarine fissure in the V1 cortical area, the lower portion of the fissure will respond to upper visual field stimuli and vice versa for the upper portion of the fissure. For this reason, and as the upper and lower portions of the fissure contain opposing dipoles, upper and lower visual field stimuli will create waveforms of opposing polarity in the EEG. B, Upper and lower visual field stimuli used for C1 VEP task are centered $3^{\circ}$ below the fixation point to account for the overrepresentation of the lower visual field in the calcerine fissure as shown in $\boldsymbol{A}$. $\boldsymbol{C}, \mathrm{C} 1 \mathrm{VEP}$ from a single participant in response to the upper and lower stimuli, the difference was measured as the lower minus the upper VEP. The peak latency of the difference wave (indicated as + ) was used as the estimate of $t 2$.

State University and the Air Force Research Laboratory. All participants were compensated for their time.

\section{Recording}

All recordings were made using the BioSemi ActiveTwo system (BioSemi B.V.). Recordings were made with a $2048-\mathrm{Hz}$ sampling rate at 64 channel locations based on the modified combinatorial nomenclature extension of the 10-10 system (American Electroencephalographic Society, 1994) excluding the inferior chain with the exception of the channels P9/P10 and Iz (Seeck et al., 2017), with bilateral electrodes on the mastoid process, infraorbital, and outer canthus locations. Participant responses were recorded using a low-latency mechanical keyboard (Cherry MX 6.0 [G80-3930], Cherry GmbH). This was combined with other task-state and visual-stimulus timing information via light sensors placed on the monitor to record events on the ActiveTwo's 16-bit trigger line (StimTracker 1G, Cedrus Corporation).

\section{Stimuli}

All tasks and stimuli were constructed and presented using MATLAB (R2011b; The MathWorks) and the Psychophysics Toolbox (v3.0.13; Brainard, 1997; Pelli, 1997; Kleiner et al., 2007). This software was run on a Dell Precision T3610 computer (Dell Inc.) with the Windows 7 Professional operating system (Microsoft Corporation). Stimuli were presented to participants on a 24.5", $240 \mathrm{~Hz}$ monitor (BenQ ZOWIE XL2540, BenQ Corporation) providing 4.2-ms temporal resolution for stimulus presentation. Where relevant, stimulus luminance was measured using a light meter (Light Meter LUXV FC 840020, SPER SCIENTIFIC). The participant's head position was fixed using a chinrest placed $58 \mathrm{~cm}$ from this monitor. The experiment was conducted in a dark room with a natural sound machine (Dohm Classic, Marpac LLC) to mask noise disturbances.

\section{Data analysis}

All data analyses were performed in MATLAB (R2019b; The MathWorks) using the EEGLAB Toolbox (v2019.0; Delorme and Makeig, 2004) and the ERPLAB plugin (v7.0.0; Lopez-Calderon and Luck, 2014). Statistical analyses were conducted using MATLAB's Statistics and Machine Learning Toolbox (R2019b; The MathWorks). This software was run on a Lenovo ThinkPad P50 computer (Lenovo) with the Windows 10 Enterprise operating system (Microsoft Corporation).

\section{Code accessibility}

Task design and data analysis code are made publicly available at https://osf.io/qnryf/; DOI 10.17605/OSF.IO/ QNRYF in addition to the recorded EEG data.

\section{Estimation of $\mathbf{t} 2$}

The peak latency of each participant's C1 VEP component was used as an estimate of their individual retina-to$V 1$ conduction delay, $t 2$. This component reflects the arrival of a volley of visual information to V1 from the LGN along the optic radiations. This is analogous to the $\alpha$ wave, in the sense that it also reflects excitatory burst volleys from the LGN to $\mathrm{V} 1$, according to the hypothesized model. The $\mathrm{C} 1$ peak, rather than onset, latency was used to estimate the middle point of these volleys rather than their onset. Because of the specific folding of the V1 cortical area around the calcarine fissure (Fig. 1A), the C1 wave shape will vary with the location of the stimulus in the visual field. Stimuli presented horizontally centered in the visual field will stimulate spatially opposing V1 neurons whose dipoles will cancel each other out, yielding no measurable C1 component in the EEG (Clark et al., 1994; Di Russo et al., 2002). This is similarly the case for stimuli presented $\sim 3^{\circ}$ below the vertical center (Clark et al., 1994; Di Russo et al., 2002). However, with some horizontal spacing, bilateral stimuli presented above $-3^{\circ}$ in the 
visual field will produce a negative $\mathrm{C} 1$ wave, while those presented below $-3^{\circ}$ in the visual field will produce a positive C1 wave as shown in Figure $1 B$ (Clark et al., 1994; Di Russo et al., 2002).

C1 waves are found to be measured maximally at electrode $\mathrm{POz}$ using an averaged mastoid reference, but they are still quite small in amplitude. To obtain a well-defined C1 VEP component, both negative and positive components were obtained using upper and lower visual field stimuli. Next, the difference between these two signals was taken to create a large-amplitude wave with a clear peak (Fig. $1 C$ shows these waveforms from a single participant). This peak latency was taken as an estimate of $t 2$.

\section{Experimental design}

The stimulus used for this task was a $17^{\circ}$ wide, $6.38^{\circ}$ tall black and white checkerboard made of $16 \times 61.06^{\circ}$ squares placed on an otherwise black screen with a fixation point placed in the center (Fig. 1B). The checkerboard was flashed with its center either $5^{\circ}$ above or $7.5^{\circ}$ below the fixation point and centered horizontally. The size and placement of these stimuli were selected to generate maximum amplitude waves (Clark et al., 1994). A total of 600 upper and 600 lower stimuli were presented in a mixed random order each with a duration of $33 \mathrm{~ms}$ with random 250 - to $450-\mathrm{ms}$ interstimulus intervals, resulting in a trial time of $\sim 7.5 \mathrm{~min}$.

Similarly to Di Russo et al. (2002), participant attention and gaze were maintained on the fixation point by a sham task performed during the trial. The fixation point would occasionally flash to a brighter color for $12.6 \mathrm{~ms}$, and the participant was instructed to watch for this flash and to respond by pressing the space bar.

\section{Analysis}

For the C1 VEP analysis, the originally recorded 2048$\mathrm{Hz}$ sampling rate was maintained but re-referenced to averaged mastoids. All EEG signals were bandpass filtered from 0.01 to $50 \mathrm{~Hz}$ at $-6 \mathrm{~dB}$, using a second order IIR Butterworth filter as implemented in the ERPLAB toolbox (Lopez-Calderon and Luck, 2014), to achieve a zerophase filter response. A bipolar vertical electrooculogram (vEOG) signal was created by subtracting the averaged left and right infraorbital electrodes from the averaged Fp1 and Fp2 electrodes.

The data were epoched from $50 \mathrm{~ms}$ before to $200 \mathrm{~ms}$ after stimulus presentation for both the upper and lower visual stimuli. Blinks within these epochs were detected by sliding a $150-\mathrm{ms}$ window at $75-\mathrm{ms}$ steps over the vEOG signal, and any window containing a peak-to-peak amplitude of $100 \mu \mathrm{V}$ or greater was rejected from analysis. Separate average VEPs were calculated for the upper and lower stimulus trials and were baseline-corrected to the 50-ms prestimulus period. The lower visual field VEP was then subtracted from the upper field VEP to create a difference wave containing a $\mathrm{C} 1$ wave with a clear peak.

The $\mathrm{C} 1$ peak latency was measured at electrode $\mathrm{POz}$ by finding the most negative peak within the 0 - to $110-\mathrm{ms}$ time window relative to stimulus onset. This peak latency

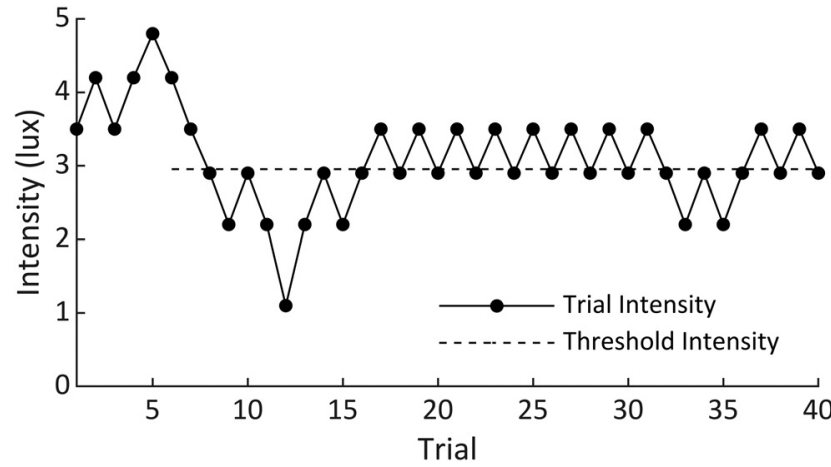

Figure 2. Example of the staircase method used to determine the threshold intensity in a single participant. Each time the participant observed the stimulus, the intensity in the next trial was decreased. If the participant did not observe the stimulus in one trial, the intensity was increased in the next trial. Threshold intensity was determined as the average intensity of all trials (trials $6-40$ in this example) following the third reversal, or corner, in the trial-intensity trace (trial 5 in this example). The dashed threshold intensity line in this figure spans the trials over which the intensities were averaged.

was calculated for each participant individually and was used as an estimate of their individual $t 2$.

\section{Measuring ORs}

This task was designed to be conducted with closed eyes to evoke more frequent (Legewie et al., 1969) and higher amplitude $\alpha$ oscillations (Barry et al., 2007). For this purpose, stimuli were designed such that they could be observed as light flashes through closed eyelids. The brightness of these flashes was designed to be near threshold intensity (where participants reported seeing $\sim 50 \%$ of the stimuli) to prevent any ceiling or floor effects on performance.

\section{Experimental design}

The stimulus used for this task was an $8.5^{\circ}$ square centered on an otherwise black ( 0.7 lux) screen. The stimulus brightness was defined by coloring the square as a greyscale value from 0 (completely black; 0.7 lux) to 255 (completely white; 89.2 lux). The stimulus was designed to be observable through the closed eyes of the participant. The brightness of the stimulus was adjusted to near the threshold intensity for each individual participant, the value at which the participant reported observing the stimulus half of the number of times it was presented. This threshold was estimated using the staircase method as described in Cornsweet (1962) during a calibration task before the main task. In this task, the participant kept their eyes closed as a series of flashes were presented. Each time the participant reported observing a flash (using the spacebar), the stimulus intensity was decreased in the next trial. However, if the participant did not report observing the stimulus, the intensity was increased on the next trial. Following the methods described in Cornsweet (1962), threshold intensity is defined as the average of all trials following the third reversal in trial-intensity slope. An example of this task from one participant is shown in 
Figure 2. In this figure, the third reversal occurred at trial 5 , and threshold intensity was calculated as the average of trials $6-40$.

The stimuli used were so dim that the participants' ability to perceive them was sensitive to how well their eyes had adapted to the dark. Pilot testing of the task revealed that, without an adjustment period, the threshold brightness found during the calibration task would become easier to see over the course of the main task. Although visual adaptation to the dark will continue over many hours, the most rapid changes occur in the first $15 \mathrm{~min}$, and visual acuity begins to plateau in 20 min (Bierings et al., 2018). Therefore, participants were given $25 \mathrm{~min}$ in the dark as an adaptation period before conducting the calibration task to estimate the threshold brightness.

In the main task, the stimuli were flashed for a period of $8.4 \mathrm{~ms}$, and the participant was instructed to respond using the keyboard any time they observed a flash. The participant had to respond with the space bar within $2 \mathrm{~s}$ of the stimulus to be a valid response. The experimenter triggered the stimuli during the times of occipital $\alpha$ oscillation as observed in the real-time data throughout the task. After the experimenter triggered the stimulus, it was presented after a random interval of $<750 \mathrm{~ms}$ (to mitigate potential confounding effects of experimenter bias in stimulus presentation timing).

\section{Analysis}

All data were down-sampled to $512 \mathrm{~Hz}$ and re-referenced to averaged mastoids. The signal was bandpass filtered from 0.01 to $50 \mathrm{~Hz}$ at $-6 \mathrm{~dB}$ using a second order IIR Butterworth filter, as implemented in the ERPLAB toolbox (Lopez-Calderon and Luck, 2014), to achieve a zerophase filter response. A bipolar vEOG signal was created by subtracting the averaged left and right infraorbital electrodes from the averaged Fp1 and Fp2 electrodes.

To isolate the activities of the left and right visual cortices, a left occipital (LO) signal was calculated by averaging the channels $01, \mathrm{PO}$, and PO7, and a right occipital (RO) signal was calculated by averaging channels $\mathrm{O} 2$, PO4, and PO8. LO and RO were then convolved with a $10-\mathrm{Hz}$ complex Morlet wavelet to obtain time-domain power and phase signals (Cohen, 2014). A peak-to-peak amplitude signal was then calculated by doubling the square root of the power signal.

The Morlet wavelet was constructed with a center frequency of $10 \mathrm{~Hz}$ containing $2 \frac{2}{3}$ cycles (the recommended minimum; Cohen, 2019). This cycle count was chosen to optimize temporal resolution and resulted in a wavelet with a full-width at half maximum (FWHM) of $101.6 \mathrm{~ms}$ in the time domain and $8.87 \mathrm{~Hz}$ in the frequency domain. A FWHM of $8.87 \mathrm{~Hz}$ indicates that the wavelet convolution was effectively a bandpass filter with half-power points of 5.57 and $14.44 \mathrm{~Hz}$, approximating the $\alpha$ frequency band.

The following analysis was then performed independently for each participant. Amplitude and phase were measured from LO and $\mathrm{RO}$ at the individuals' $t 2$ relative to stimulus onset for both observed and missed trials. Trials in which LO and RO phases differed by $>90^{\circ}$ were rejected from further analysis to control for $\alpha$ asynchrony between the hemispheres. For each of the remaining trials, a single amplitude and phase value was obtained by averaging the amplitude and phase of the LO and RO signals. Phase values were averaged using appropriate statistics for circular quantities (Berens, 2009). These trials were then divided by median amplitude into high-amplitude and low-amplitude bins and then further divided by phase using $90^{\circ}$ bins centered on $0^{\circ}, 90^{\circ}, 180^{\circ}$, and $270^{\circ}$ phase angles. The amplitude median was chosen to provide equal sample sizes in each amplitude bin, and since it is appropriate regardless of distribution normality. When the individual amplitude distributions of our 20 subjects were examined, they appeared to be unimodal and approximately normal with no point of division appearing to be more advantageous than the median (Fig. 3). ORs (the percentage of trials in which the participant observed the stimulus) were then calculated for every bin, and the overall OR across all bins (i.e., the average OR over all trials) was subtracted from these values to obtain a $\Delta O R$ value for each of the two (amplitude) $\times$ four (phase) bins. Note that $\triangle O R$ is not a measure of percent change, but simply a difference of the overall OR and the conditionspecific OR.

\section{Statistical analysis}

This analysis resulted in a within-subjects $2 \times 4$ repeated measures design with 20 participants and two factors: amplitude (high, low) and phase $\left(0^{\circ}, 90^{\circ}, 180^{\circ}, 270^{\circ}\right)$. These data were analyzed using a repeated measures ANOVA, testing both main effect of amplitude and phase as well as their interaction. Independent $t$ test and Tukey's HSD tests were used to probe any effects found to be significant. All statistical analyses were conducted using MATLAB's Statistics and Machine Learning Toolbox (R2019b; The MathWorks).

\section{Results}

\section{Individual retina-to-V1 conduction delay (t2) measurement}

In the first part of the experiment, the goal was to estimate each participant's retina-to-V1 conduction delay, $t 2$. To calculate that, we measured in each participant the averaged VEPs in response to upper and lower visual stimuli and calculated the "difference waveform" (based on the difference between two signals); the peak latency of this difference waveform was taken as the participant's t2 (Fig. 4). The mean t2 for all participants was $75.56 \mathrm{~ms}$ [range $=[62.99,88.87] \mathrm{ms}, 95 \%$ confidence interval $(\mathrm{Cl})=$ $[72.44,78.69] \mathrm{ms}]$. These data indicate that it takes, on average, $75.56 \mathrm{~ms}$ for visual information to be transmitted from the retina to area V1. For each participant, the phase and amplitude measurements were made at the individual's $t 2$ relative to the stimulus onset to assess visual sensitivity.

\section{OR measurement}

The second part of the experiment aimed to investigate whether perceptual performance, when measured at $t 2$, is higher at an $\alpha$ wave trough (i.e., phase angle of $270^{\circ}$ ) than an $\alpha$ wave peak (i.e., phase angle of $90^{\circ}$ ). To examine this, 

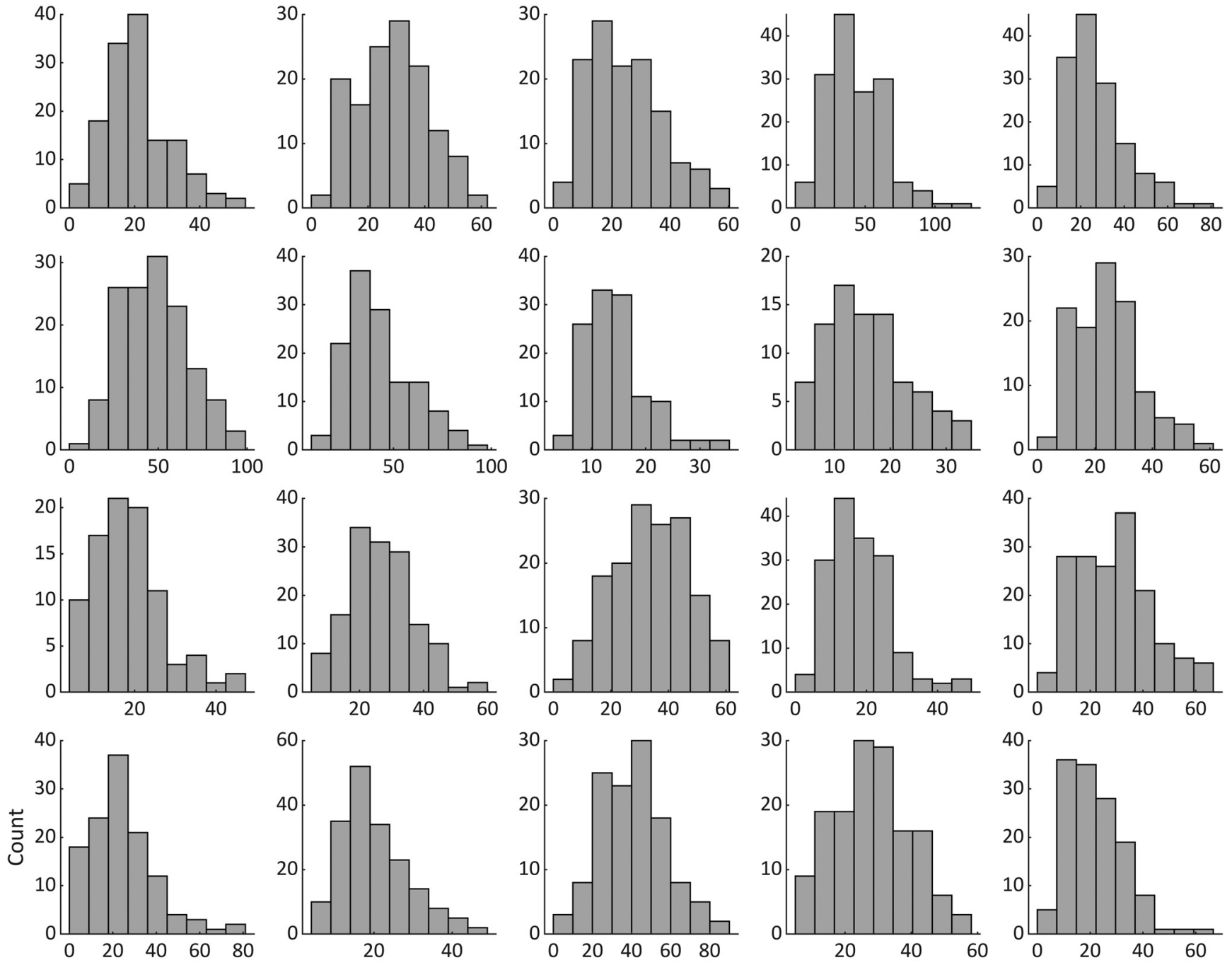

Figure 3. Each of the 20 participants' $\alpha$ peak-to-peak amplitude distribution for all trials used in the analysis at $t 2$.

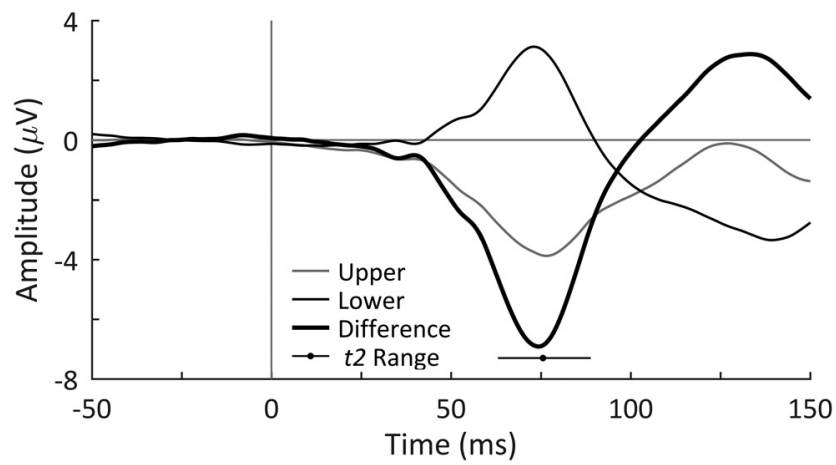

Figure 4. C1 VEP component averaged across all participants in response to upper and lower visual field stimuli. The difference waveform is the lower waveform subtracted from the upper waveform. The mean and range of each participant's estimated retina-to-V1 conduction delay ( $t 2)$ is indicated and was calculated as the peak latency of the $\mathrm{C} 1$ component in each individual's difference waveform. we presented light flashes to each participant through closed eyes. Light flashes were near individual threshold intensity estimated using the staircase method (Cornsweet, 1962) during a calibration task before the main task to prevent ceiling and floor performance effects. This resulted in the stimuli of average luminance across participants of 6.48 lux. Use of these near-threshold stimuli resulted in total ORs averaging $58.28 \%$ across participants.

On average, participants were presented 161.65 stimuli during the main task (range $=[120,200]$ ) with an average interstimulus interval of $12.52 \mathrm{~s}$. LO and RO amplitude and phase signals were measured for each participant and analyzed at the participant's t2 time point. Trials in which LO and RO phases differed by $>90^{\circ}$ were rejected from further analysis resulting in 135.75 trials remaining per participant on average (range $=[85,183]$ ). The amplitude measures were split by their median value into two bins (high and low), and the phase measures were split into four $90^{\circ}$ bins centered on $0^{\circ}, 90^{\circ}, 180^{\circ}$, and $270^{\circ}$ phase angles. A within-subjects two (amplitude) $\times$ four (phase) repeated measures ANOVA analysis was conducted 
Table 1: Two (amplitude) $\times$ four (phase) within-subjects repeated measures ANOVA results

\begin{tabular}{lllll}
\hline Source & Statistic & $p$ & $\eta_{\mathrm{p}}^{2}$ & Power \\
\hline Phase & $F_{(3,57)}=7.971$ & 0.0002 & 0.2955 & 0.986 \\
Amplitude & $F_{(1,19)}=1.751$ & 0.2014 & 0.0844 & 0.242 \\
Phase $\times$ amplitude & $F_{(3,57)}=3.770$ & 0.0154 & 0.1656 & 0.786
\end{tabular}

on the participant $\Delta \mathrm{OR}$ values (calculated as the OR per bin minus the overall OR). Mauchly tests indicated no significant violation in the assumption of sphericity for phase $\left(\chi^{2}(5)=10.169, p=0.0706\right)$, or the interaction of phase and amplitude $\left(\chi^{2}(5)=3.250, p=0.6615\right)$. The ANOVA analysis showed that phase and the interaction between phase and amplitude have statistically significant effects on ORs, as shown in Table 1. Accordingly, these ANOVA results support our hypothesis that a relationship between $\alpha$ phase and visual observation exists, and that this relationship is modulated by the $\alpha$ wave amplitude.

Multiple comparison tests were performed to investigate the precise nature of the effects found to be significant in the ANOVA. Figure 5 shows the results of independent $t$ tests (significance indicated by \#) comparing $\Delta \mathrm{OR}$ to zero in each of the phase and amplitude levels. Only phase bins $90^{\circ}$ and $270^{\circ}$ in the high-amplitude condition were found to be significantly different $\left(t_{(19)}=\right.$ -5.632, $p=0.0002 ; t_{(19)}=3.466, p=0.0207$, respectively, Bonferroni corrected for eight comparisons; Bland and Altman, 1995). Tukey's HSD tests (indicated by *) then compared $\triangle \mathrm{OR}$ distributions between phase bins within each amplitude level. These tests found significant differences in the phase bins only in the high-amplitude condition. Specifically, the $90^{\circ}$ phase bin was statistically different from the $0^{\circ}(p=0.0077), 180^{\circ}(p=0.0308)$, and $270^{\circ}(p<0.0001)$ phase bins in the high-amplitude condition (Fig. 5). Importantly, the greatest difference in the high-amplitude condition was found between phase bins $90^{\circ}$ and $270^{\circ}$, with a $22.20 \%$ increase in ORs $(95 \% \mathrm{Cl}=$ [9.77\%, 34.63\%]; Fig. 5). Collectively, these results show that stimuli are observed with greatest probability when the $\alpha$ wave is near a trough at $t 2$, and with lowest probably when near a peak, when $\alpha$ amplitude is high. The perception-phase relationship is robust only at high $\alpha$ wave amplitude, likely because during low-amplitude oscillations the measured phase is less representative of the underlying neural population activity.

\section{Phase-varying analysis of visual observation}

For a high-resolution characterization of the phase effect on $\Delta \mathrm{OR}$, we repeated the original procedure at $t 2$ using $90^{\circ}$ phase bins rotated in $1^{\circ}$ increments for all $360^{\circ}$ phase angles. Figure 6 shows $\Delta \mathrm{OR}$ for each of the 20 participants in both the high-amplitude and low-amplitude conditions as a function of the centered $\alpha$ phase angle of the bins. Indicated along the circumference is the direction of the center of mass of these phase diagrams for each condition, calculated as the angle of the vector mean of each of the 360 points forming the $\Delta$ OR distribution. This angle is referred to here as the preferred phase and indicates the phase at which stimuli have the highest probability of being observed given the whole distribution. Note that in the high-amplitude condition, the preferred phase is between $180^{\circ}$ and $360^{\circ}$ (the negative-amplitude portion of the $\alpha$ cycle) in 18/20 participants. These preferred phase angles have associated magnitudes indicating how far the distribution's center of mass is offset in that direction and the strength of the effect of phase on ORs. These preferred phase angles and magnitudes for all participants are shown in Figure $7 B, D$.

Figure $7 A, C$ shows the $\triangle \mathrm{OR}$ distributions averaged across all participants. Indicated in black along the circumference is the group's preferred phase. Note that calculating the group's preferred phase as the direction to the center of mass of the group's $\Delta O R$ distributions (Fig. $7 A, C$ ) is equivalent to calculating the vector mean of the 20 individual preferred phases and associated magnitudes (Fig. $7 B, D$ ). By taking the $\triangle O R$ value at the preferred phase angle and subtracting from it the corresponding opposite phase angle (by $180^{\circ}$, referred to as the pessimal phase), we calculated the preferred phase effect (PPE), which indicates the increase in OR from pessimal to preferred $\alpha$ phase angles, relative to t2. Our results showed that the preferred phase was similar in both the high-amplitude and low-amplitude conditions $\left(272.41^{\circ}\right.$ and $284.65^{\circ}$, respectively) with a PPE of $20.96 \%$ in the highamplitude condition but $9.12 \%$ in the low-amplitude condition. These data further substantiate an interaction effect on observation probability between $\alpha$ wave phase and amplitude, which was observed in the prior ANOVA analysis.

Additionally, we estimated the 95\% Cls for the group's preferred phase, which are shown as the shaded regions along the circumference of the phase diagrams in Figure 7. These Cls could only be estimated since there is not a known method of computing an angular $\mathrm{Cl}$ on non-unit vectors directly. To estimate these Cls, the 20 individual preferred phase vectors (with associated magnitudes) were first converted to cartesian values. Then, two $95 \%$

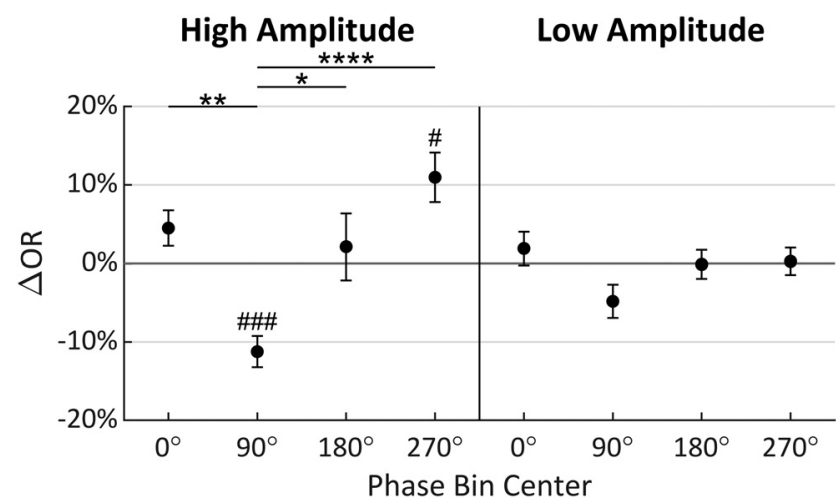

Figure 5. Mean change in $\mathrm{OR}(\triangle \mathrm{OR})$ for each amplitude and phase condition relative to mean OR across all conditions when analysis was conducted at $t 2$ (error bars indicate standard error). Tukey's HSD tests were performed between phase levels within amplitude conditions with significance indicated by $*$. Bonferroni corrected $t$ tests compared each mean to zero with significance indicated by $\# ; 90^{\circ}=$ peak; $* \# p \leq 0.05 ; * *$, $\# \# p \leq 0.01 ; * * *, \# \# \# p \leq 0.001 ; * * * *, \# \# \# \# p \leq 0.0001$. 

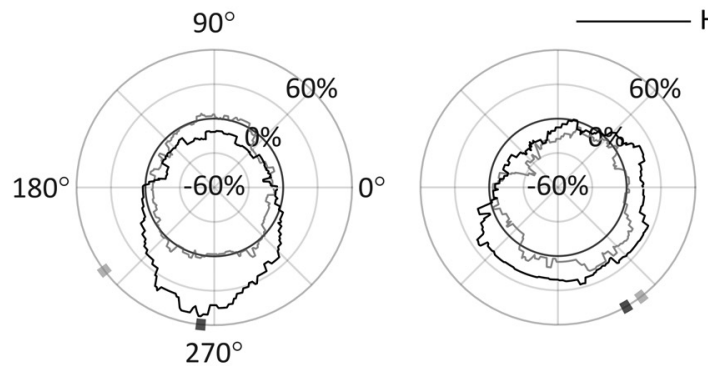

High Amplitude Low Amplitude
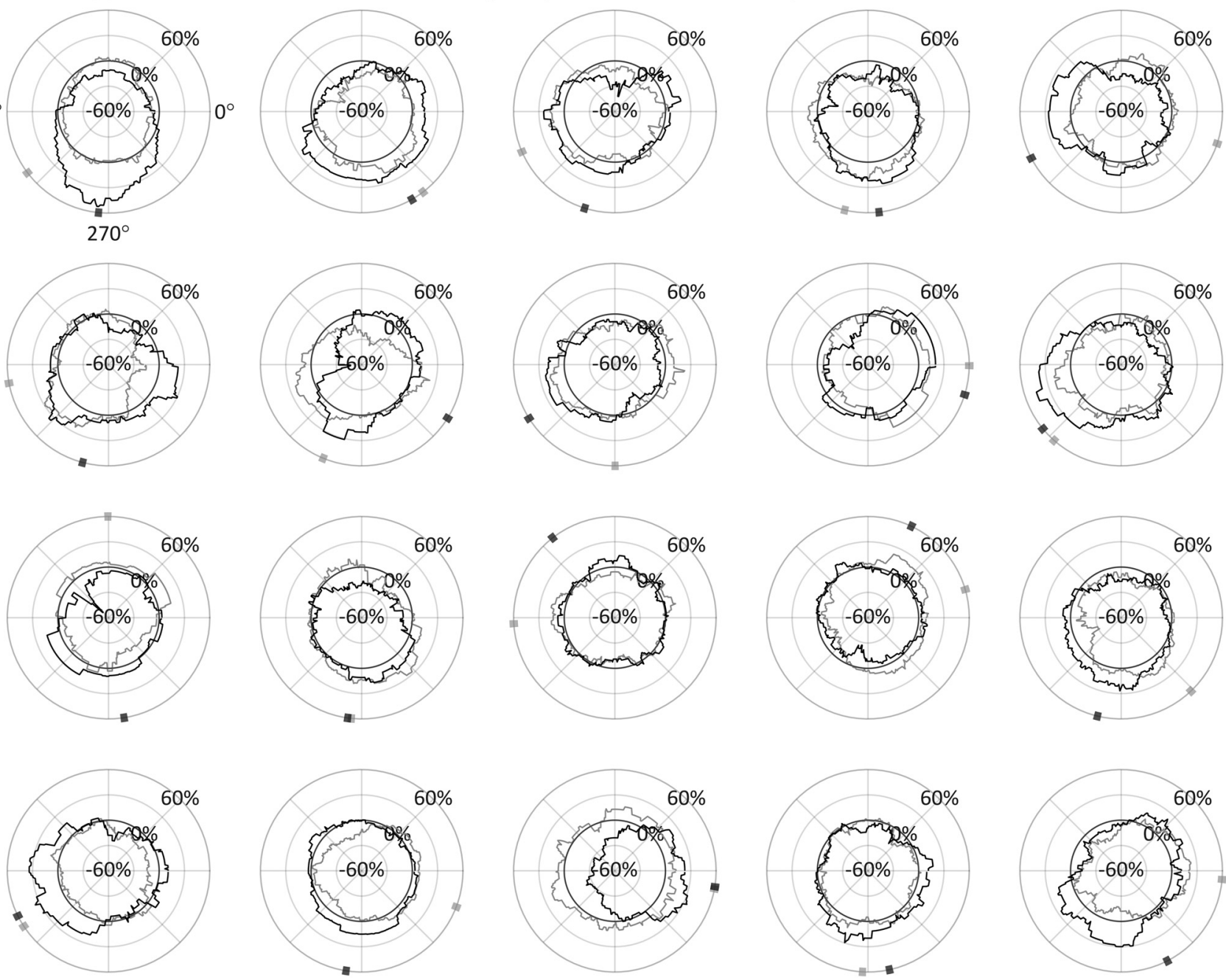

Figure 6. $\triangle \mathrm{OR}$ as a function of phase for each participant in the high-amplitude and low-amplitude conditions with radial axis extending to negative values for analysis conducted at $t 2$. The preferred phase is indicated on the circumference, calculated as the direction toward the circle's center of mass; $90^{\circ}=$ peak.

\section{High Amplitude}

A

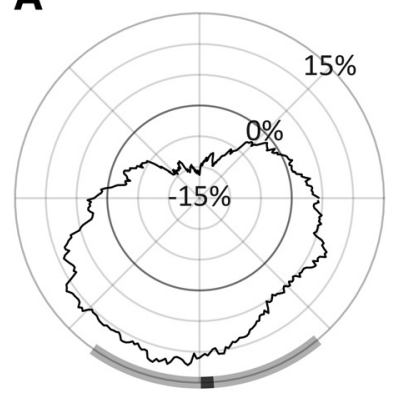

B

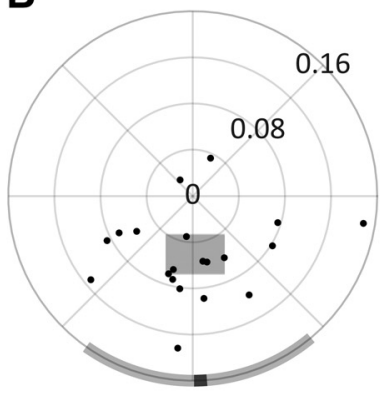

Low Amplitude

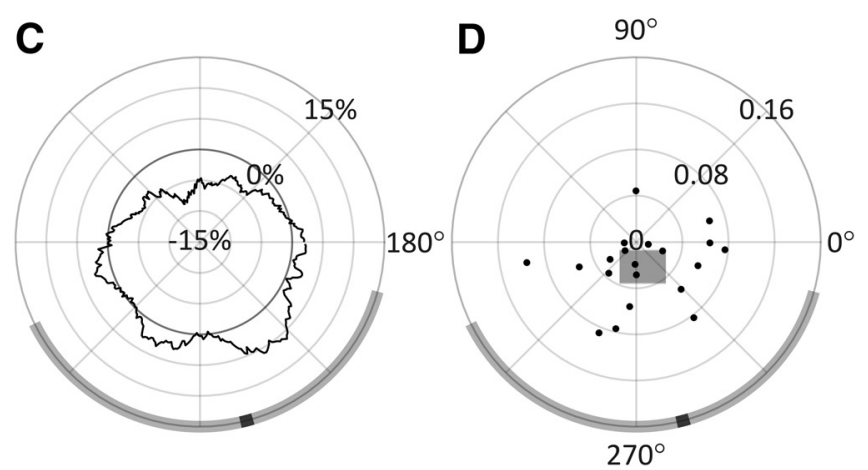

Figure 7. Analysis conducted at $t 2 . \boldsymbol{A}, \boldsymbol{C}, \Delta \mathrm{OR}$ as a function of phase, with radial axis extending to negative values. $\boldsymbol{B}, \boldsymbol{D}$, Individual participants' preferred phase angles; their magnitude is proportional their individual PPE. The width and height of the shaded rectangles indicate, respectively, the $x$ and $y 95 \% \mathrm{Cl}$ in cartesian space. In all panels, the group mean preferred phase is indicated in black on the circumference of the diagrams, with the shaded region indicating estimated $95 \%$ Cls for each amplitude condition; $90^{\circ}=$ peak. 
A

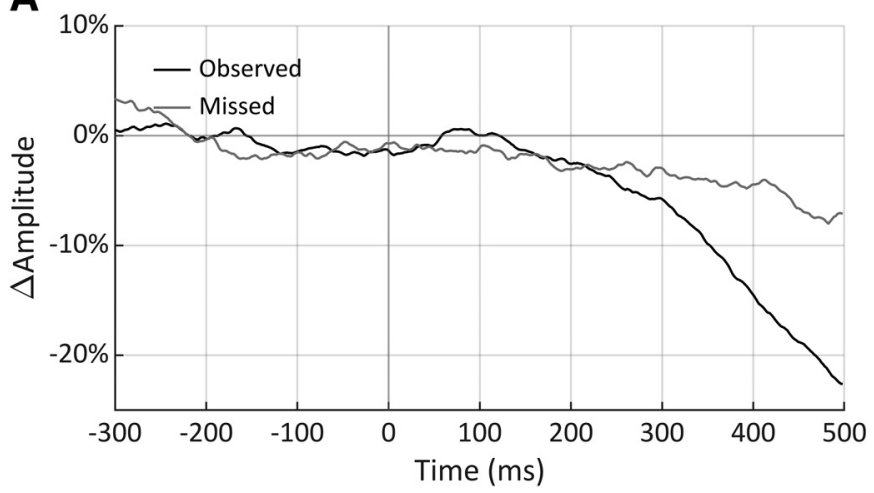

B

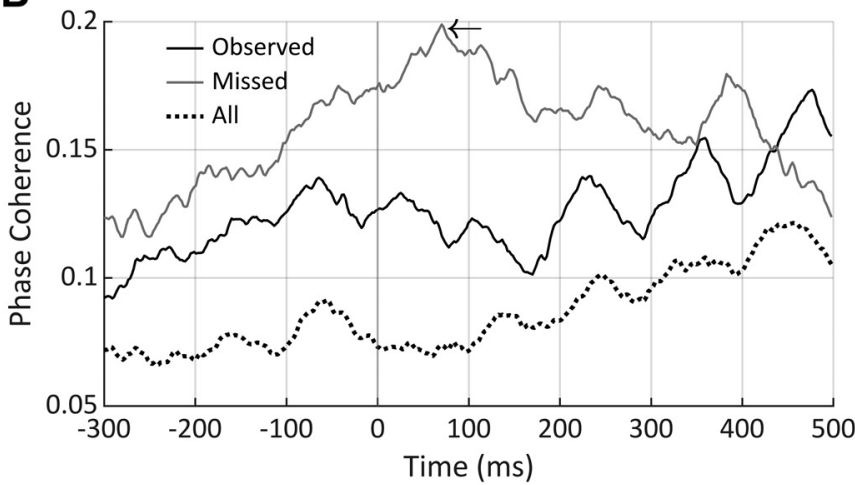

Figure 8. Change in $\alpha$ amplitude, relative to a -300 to $-100 \mathrm{~ms}$ baseline interval $(\boldsymbol{A})$, and $\alpha$ phase coherence for observed, missed, and all stimuli together $(\boldsymbol{B})$. The arrow indicates a peak in the missed trial phase coherence reflecting the time point were $\alpha$ phase best predicts that a trial will be unobserved by the participant. There was no indication of stimulus-induced effects at the $t 2$ time point where phase and amplitude measures were hypothesized to be of mechanistic relevance.

Cls were calculated separately on both the $x$ and $y$ components, indicated, respectively, as the width and height of the shaded rectangles in Figure $7 B, D$. The angular interval that encompassed both Cls was then used to represent the estimated $95 \% \mathrm{Cl}$ on the preferred phase in the polar space, although this interval will not necessarily be centered on the preferred phase (i.e., mean). In the highamplitude condition, this resulted in an estimated $95 \% \mathrm{Cl}$ of $\left[234.75^{\circ}, 309.84^{\circ}\right]$, and in the low-amplitude condition a much wider $\mathrm{Cl}$ of $\left[206.22^{\circ}, 344.45^{\circ}\right]$. Together with Figure $7 A, B$, these results show that observations rates are highest at the trough of the $\alpha$ wave, and lowest at the peak. This effect is prominent in the high-amplitude condition, but also to a much lesser extent in the low-amplitude condition recalling these effects did not reach significance in the prior ANOVA.

\section{Measuring induced activity}

$\alpha$ Band desynchronization (ERD) and phase resetting are characteristically observed in response to relevant visual stimuli (Klimesch et al., 2011). These reflect neural processes resulting from the stimulus, not spontaneous activity, and so in our case are only expected in response to observed trials, not missed trials. To ensure that the amplitude and phase measurements made here are the result of spontaneous brain activity and not caused by the stimulus itself, we verified that a drop in amplitude or sudden change in phase coherence across trials does not occur in the observed trials at $t 2$. Thus, we first calculated the percent change in $\alpha$ amplitude, relative to a prestimulus baseline period of -300 to $-100 \mathrm{~ms}$, for both the observed and missed trials and these are shown in Figure $8 A$. There was the expected characteristic drop in $\alpha$ amplitude (i.e., ERD) in response to the observed stimuli; however, the this was not observed until after $100 \mathrm{~ms}$ poststimulus, which did not drop below that of the missed stimuli until after $200 \mathrm{~ms}$. This result indicates that measurements made at $t 2$ (whose mean value is $75.56 \mathrm{~ms}$ and range $=[62.99,88.87] \mathrm{ms})$ were safe from stimulus induced amplitude effects.
To ensure that there was no event related phase resetting, we next calculated phase coherence across all trials, as well as just the observed and missed trials (Fig. 8B). As shown in Figure $8 B$, no phase resetting was apparent in the observed trials or in all trials together, especially before $150 \mathrm{~ms}$. No event related phase resetting would be expected in the missed trials, and this was not seen. However, in the phase coherence across missed trials, there is a prominent peak measured at the 70.31-ms time point, marked by the arrow in Figure $8 B$, but this not an event related phase reset as will be described further in Discussion. In sum, Figure 8 confirmed that no event related amplitude change or phase reset occurred before $100 \mathrm{~ms}$ poststimulus, demonstrating that the phase and amplitude measurements taken in the present study are not the result of stimulus induced activity but of ongoing spontaneous oscillations.

\section{t2 measurements versus prestimulus measurements}

To evaluate the benefit of using the individual $t 2$ time point for measuring the $\alpha$ phase effect on perceptual performance, we repeated the above analysis at a time point $100 \mathrm{~ms}$ before stimulus onset $(t=-100 \mathrm{~ms}$ ) and compared the results to those obtained above at $t 2$. These measurements have been taken in previous studies in prestimulus periods or at stimulus onset to avoid measuring induced oscillatory effects as mentioned above (Mathewson et al., 2009, 2012; Milton and Pleydell-Pearce, 2016; Kizuk and Mathewson, 2017). Whereas in the previous analysis, the four phase bins centered at $0^{\circ}, 90^{\circ}, 180^{\circ}$, and $270^{\circ}$ were chosen a priori based on their theoretical significance at the 2 time point, no prediction could be made about the relevant phase bins at this prestimulus time point. Instead, the analysis was first performed in $90^{\circ}$ phase bins in $1^{\circ}$ increments, as was performed previously (i.e., in Fig. 7), and the four relevant phase bins were chosen empirically based on the observed preferred phase in the high-amplitude condition. In other words, the phase bins were chosen post hoc to reveal the highest effect phase has on amplitude for this prestimulus $t=-100 \mathrm{~ms}$ time point. 


\section{High Amplitude}

A

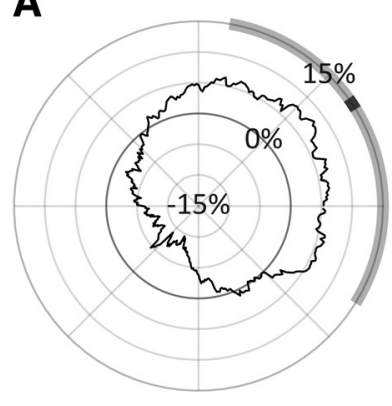

B

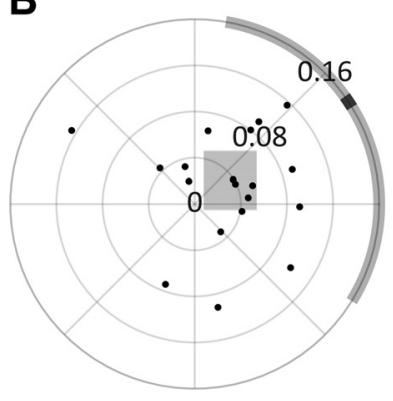

Low Amplitude

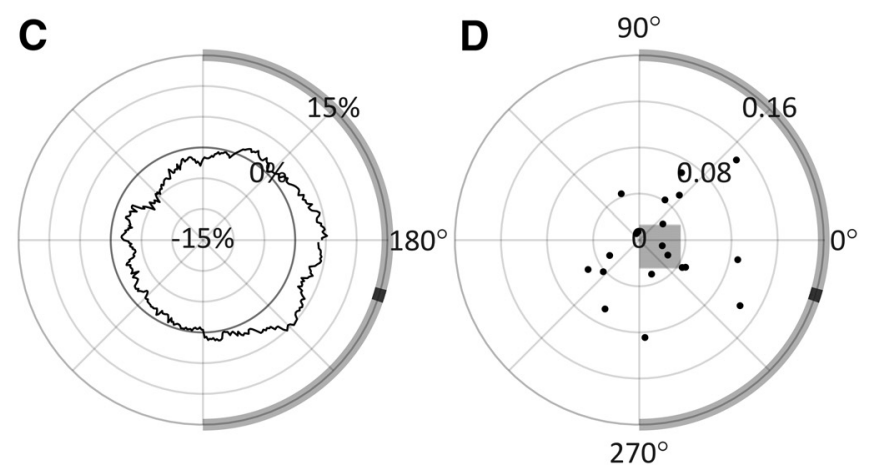

Figure 9. Analysis conducted at $t=-100 \mathrm{~ms}$. A, C, $\Delta \mathrm{OR}$ as a function of phase, with radial axis extending to negative values. $\boldsymbol{B}, \boldsymbol{D}$, Individual participants' preferred phase angles; their magnitude is proportional their individual PPE. The width and height of the shaded rectangles indicate, respectively, the $x$ and $y 95 \% \mathrm{Cl}$ in cartesian space. In all panels, the group mean preferred phase is indicated in black on the circumference of the diagrams, with the shaded region indicating estimated $95 \% \mathrm{Cls}$ for each amplitude condition; $90^{\circ}=$ peak.

The results of this analysis performed over all $90^{\circ}$ bins in both the high-amplitude and low-amplitude conditions are shown in Figure 9. In the high-amplitude condition, a phase effect on perceptual performance can again be observed, however in this case the preferred phase was measured at $33.53^{\circ}\left(95 \% \mathrm{Cl}=\left[-31.69^{\circ}, 80.29^{\circ}\right]\right)$ with a $15.48 \%$ PPE. In the low-amplitude condition, the preferred phase was measured at $-17.22^{\circ}(95 \% \mathrm{Cl}=$ $\left.\left[-90.04^{\circ}, 90.07^{\circ}\right]\right)$ with a $5.22 \%$ PPE. Based on the preferred phase measured in the high-amplitude condition for prestimulus $t=-100 \mathrm{~ms}$, the four $90^{\circ}$ phase bins centered at $33^{\circ}, 123^{\circ}, 213^{\circ}$, and $303^{\circ}$ were therefore chosen for the $2 \times 4$ repeated measures ANOVA analysis. Mauchly tests indicated no significant violation in the assumption of sphericity for phase $\left(\chi^{2}(5)=5.194, p=\right.$ $0.3926)$, or the interaction of phase and amplitude $\left(\chi^{2}(5)=8.861, p=0.1147\right)$. As shown in Table 2, only the main effect of phase was found to have a statistically significant effect on ORs. Despite these phase bins showing the highest phase effects at $t=-100 \mathrm{~ms}$, phase, whose bins were chosen a priori based on theoretical significance, had a stronger effect on observation when the measurements were taken at $t 2$ (with roughly twice the effect size).

Figure 10 shows the multiple comparisons result for this data collected at $100 \mathrm{~ms}$ before stimulus onset for better comparison to those from t2 shown in Figure 5. Bonferroni corrected independent $t$ tests compared each $\Delta \mathrm{OR}$ value to zero, but none were found to be significant, indicating that none of the ORs in any of these four phase bins differed from the overall mean OR either amplitude

Table 2: Two (amplitude) $\times$ four (phase) within-subjects repeated measures ANOVA results repeated at $100 \mathrm{~ms}$ before stimulus onset

\begin{tabular}{lllll}
\hline Source & Statistic & $p$ & $\eta_{0}^{2}$ & Power \\
\hline Phase & $F_{(3,57)}=3.378$ & 0.0243 & 0.1510 & 0.735 \\
Amplitude & $F_{(1,19)}=0.008$ & 0.9289 & 0.0004 & 0.051 \\
Phase $\times$ amplitude & $F_{(3,57)}=1.695$ & 0.1784 & 0.0819 & 0.420
\end{tabular}

condition. Tukey's HSD tests (indicated by *) were used to compare $\triangle \mathrm{OR}$ distributions between phase bins within each amplitude level, but these tests only found a significant difference between $33^{\circ}$ and $213^{\circ}(p=0.0143)$, where there was a $15.47 \%$ difference in observations rates $(95 \%$ $\mathrm{Cl}=[2.39 \%, 28.55 \%])$.

In our original hypothesis, we predicted that the highamplitude preferred phase would be $270^{\circ}$ at $t 2$, which was approximately $t=75 \mathrm{~ms}$ on average. Assuming (1) a perfectly stationary and (2) an exactly $10-\mathrm{Hz}$ signal, we would have predicted that the preferred phase at $t=-100$ $\mathrm{ms}$, as well as at $t 0$, would have been $0^{\circ}$. This was approximately what was found here; however, the inexactness is expected since neither assumption is exactly met in the natural $\alpha$ rhythm. The non-stationarity would additionally explain the decrease in effect size from $t 2$ to $t=-100 \mathrm{~ms}$. Collectively, these results show that although an $\alpha$ phase effect on ORs in a prestimulus period can be observed, the strength of the effect is much weaker than when

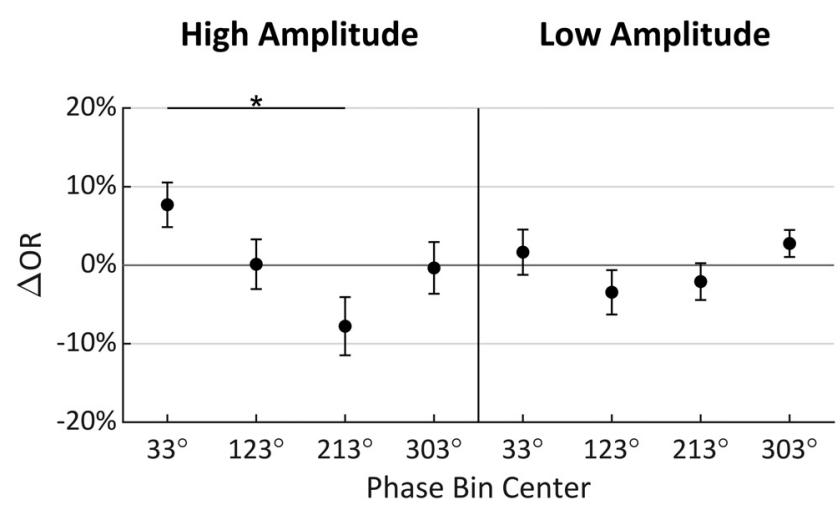

Figure 10. Mean change in $\mathrm{OR}(\triangle \mathrm{OR})$ for each amplitude and phase condition relative to mean OR across all conditions when analysis was conducted at $t=-100 \mathrm{~ms}$ (error bars indicate standard error). Tukey's HSD tests were performed between phase levels within amplitude conditions with significance indicated by $*$. Bonferroni corrected did not find any of the means to significantly differ from zero; $90^{\circ}=$ peak; $* p \leq 0.05$. 
measurements are taken at $t 2$. This finding shows that measurement of $\alpha$ oscillation amplitude and phase have a stronger correlation with visual perception at $t 2$ than at $t=$ $-100 \mathrm{~ms}$.

\section{Discussion}

This study presents a novel, controlled method for examining the neuronic shutter effect, hypothesized to be reflected in the posterior $\alpha$ oscillations observed in human EEG. Our hypothesis was that perceptual performance is modulated by the $\alpha$ rhythm in a precise phase and timing relationship. To test this relationship, we (1) presented near-threshold intensity visual stimuli through closed eyes to participants; (2) accounted for individual retina-to-V1 conduction delays (t2); (3) controlled for asynchrony between hemispheres; and (4) measured the $\alpha$ phase and amplitude relative to $t 2$. Our results confirm the hypothesized relationship between the $\alpha$ rhythm phase and perception, with the greatest rates of observation occurring at the $\alpha$ wave trough (excitatory state) and the lowest rates at the $\alpha$ wave peak (inhibitory state). The perception-phase relationship appears to be modulated by $\alpha$ amplitude as it is observed at high, but not low, $\alpha$ wave amplitude. This work is novel in considering the underlying neural structure and function of the visual system when predicting the exact phase relationship to perception, and in controlling for individual neural conduction delay and asynchrony between hemispheres.

While the $\alpha$ wave shutter effect has been previously examined (Dustman and Beck, 1965; Mathewson et al., 2009, 2012; Milton and Pleydell-Pearce, 2016; Kizuk and Mathewson, 2017), this is the first study, to our knowledge, to directly examine the relationship between perceptual performance and spontaneous $\alpha$ phase as measured relative to each individual's conduction delay. Previous studies have either measured $\alpha$ phase at stimulus onset, to, or during prestimulus periods (Mathewson et al., 2009, 2012; Milton and Pleydell-Pearce, 2016; Kizuk and Mathewson, 2017). Accordingly, the phase measurements were not always well aligned with the LGN excitability state according to the proposed cellular mechanism. Here, when we accounted for each individual's conduction delay, as opposed to the group average conduction delay or measuring at stimulus onset $t 0$, we demonstrated the existence of a hypothesized relationship between the $\alpha$ phase and perception. Notably, these results provide the most robust evidence consistent with cyclic LGN excitability as the cellular mechanism mediating this effect, although we do not directly examine observe this mechanism. Dustman and Beck (1965) measured reaction times, as opposed to ORs, at $\alpha$ phase adjusted to group average, as opposed to individual conduction delay. This study, similar to ours, found slowest reaction times at the peak $\left(90^{\circ}\right)$ of the $\alpha$ wave, and fastest reaction times close to the $\alpha$ trough $\left(240^{\circ}\right)$.

A $2 \times 4$ repeated measures ANOVA found that $\alpha$ phase at $t 2 \mathrm{had}$ a significant effect on perceptual performance as hypothesized. The interaction effect of phase and amplitude was also found to be significant; the multiple comparisons tests in Figure 5 show that low amplitude attenuates the phase effect. This supports the hypothesis that low-amplitude $\alpha$ waves reflect asynchronous inhibition within the visual system. Although not part of our original hypothesis, it is somewhat surprising that no main effect of amplitude was found since increased $\alpha$ power has sometimes been found to predict poorer perceptual performance (Ergenoglu et al., 2004; Mathewson et al., 2009; Brüers and VanRullen, 2018). This perceptionpower relationship is usually attributed to more generalized early visual inhibition as evidenced by the attenuation of early (C1 and N150) VEP components with increased $\alpha$ power (lemi et al., 2019). Figure 7 gives a higher phase angle resolution view of the effects seen in Figure 5. In both the high-amplitude and low-amplitude conditions, the preferred phase was near the wave trough $\left(272.41^{\circ}\right.$ and $284.65^{\circ}$ at high amplitude and low amplitude, respectively). However, in the low-amplitude condition, the 95\% $\mathrm{Cl}$ of the preferred phase was much wider, and the PPE was lower $(20.96 \%$ and $9.12 \%$ at high and low amplitude, respectively). These results show how $\alpha$ amplitude modulates the effect of $\alpha$ phase on perception.

To demonstrate the significance of taking $\alpha$ amplitude and phase measurements at $t 2$, we repeated our analysis at $t=-100 \mathrm{~ms}$ and compared them to those obtained at t2. This prestimilus time point was chosen for two reasons. First, given the risk in poststimulus measurements getting altered by stimulus processing, a prestimilus time point is, therefore, "safer" than poststimulus or stimulusonset time points. Second, given that $\alpha$ oscillations have a primary frequency of $10 \mathrm{~Hz}$ (i.e., has a period of $100 \mathrm{~ms}$ ), the amplitude-phase relationship would repeat roughly every $100 \mathrm{~ms}$. Thus, our results at $t=-100 \mathrm{~ms}$ could help to predict this relationship at stimulus onset, which is $100 \mathrm{~ms}$ (or one period) away but under safer signal analysis conditions. At prestimulus time point of $-100 \mathrm{~ms}(t=$ $-100 \mathrm{~ms}$ ), we did find a significant phase effect, but by using different phase bins more relevant to that time point (i.e., $33^{\circ}, 123^{\circ}, 213^{\circ}, 360^{\circ}$ ). These phase bins were chosen post hoc to reveal the highest effect phase has on observation for this prestimulus time point. Despite showing the highest phase effects, a phase whose bins were chosen a priori based on theoretical significance had stronger effects on observation when the measurements were taken at $t 2$. Specifically, the new analysis shows that while phase was found to have a statistically significant effect on ORs between the preferred and pessimal phase bins in the high-amplitude condition, the effect size was weaker and roughly half of that at $t 2$. These results show the advantage of making phase and amplitude measurements at $t 2$ rather than in prestimulus periods.

Poststimulus measures of ongoing spontaneous oscillations are generally avoided since there is a risk that they will be altered by stimulus processing. We verified that our $t 2$ amplitude and phase measurements were not influenced by that by measuring $\alpha$ band ERD and phase coherence over the -300 - to $500-\mathrm{ms}$ time range relative to stimulus onset (Fig. 8). No stimulus related ERD or phase coherence were observed before $100 \mathrm{~ms}$ indicating that our measures at $t 2$ were independent of stimulus processing responses and instead reflected spontaneous activity. Interestingly, there was an overall increase in phase 
coherence values of the observed and missed trials compared with that of all trials combined (Fig. 8B). This increase is expected since, according to the phase effects on ORs found above, trials that are observed and missed will predominantly occupy specific, opposing phase ranges; thereby resulting in relatively high phase coherence across trials when grouped by observational outcome, and low phase coherence when grouped together. It is also important to note that the missed trial phase coherence trace features a prominent peak at $70.41 \mathrm{~ms}$ (Fig. $8 B$, arrow). We do not interpret this peak as an event related phase reset since (1) it is the peak of an upward trend beginning at least $300 \mathrm{~ms}$ before stimulus onset, (2) a decreasing trend is observed immediately afterward, and (3) no phase reset would be expected in response to an unobserved stimulus. Instead, we interpret this peak as marking the time where phase most accurately predicts that a stimulus will not be observed, which, in support of our hypothesis, is within $5 \mathrm{~ms}$ of the average estimated $t 2(75.56 \mathrm{~ms})$, the point hypothesized to best predict observational outcome.

\section{Neural mechanism}

Understanding the biological mechanism underlying $\alpha$ oscillation is important for explaining its function. Although simultaneous recordings with $\mathrm{fMRI}$ and PET correlated $\alpha$ activity with fluctuations in the thalamus, thus suggesting this might be the origin of $\alpha$ rhythm (Hughes et al., 2004; Omata et al., 2013), so far the precise mechanism in humans is not yet known. However, sleep spindles, a $\sim 10 \mathrm{~Hz} \alpha$-like oscillation that occurs in 1- to 3-s bursts during transition to sleep, do have a well-established mechanism. The sleep spindle mechanism includes a negative feedback loop between the LGN and the reticular nucleus (RN; Fig. 11). Both nuclei have T-type $\mathrm{Ca}^{+}$ channels that, when activated, exhibit burst firing. Between each burst of the LGN, the $\mathrm{Ca}^{+}$channels of the LGN are known to enter a refractory period, and the RN sends a similar, but inhibitory, burst to the LGN. During this time, sensory information from the optic nerve would be less likely to be relayed to V1. Importantly, the refractory periods of these T-type $\mathrm{Ca}^{+}$channels are known to result in each nucleus firing at a $\sim 10-\mathrm{Hz}$ rate (Lopes da Silva, 1991; Sherman, 2001; Timofeev and Bazhenov, 2005; Alexander et al., 2006; Timofeev and Chauvette, 2011). Each LGN burst firing generates massive EPSPs in V1, measured as a negativity in the occipital EEG (Timofeev and Bazhenov, 2005) and producing the $\sim 10-\mathrm{Hz}$ oscillation of sleep spindles seen on EEG. Chen et al. (2016) showed that, in mice, very similar mechanisms give rise to both sleep spindles and waking $\alpha$ oscillations and that the mechanism is capable of phasically modulating sensory transfer through the thalamus, and on this basis, they predicted the perceptual results found here. In cats, this mechanism was also found to produce waking $\alpha$ oscillations with the same phasic sensory gating effect (Lorincz et al., 2009). Although additional and alternate sources, including extrastriate cortical sources, have been proposed to give rise to this oscillatory activity (Steriade et al., 1990; Connors and Amitai, 1997; Bollimunta et al., 2008, 2011; van Dijk et al., 2008),

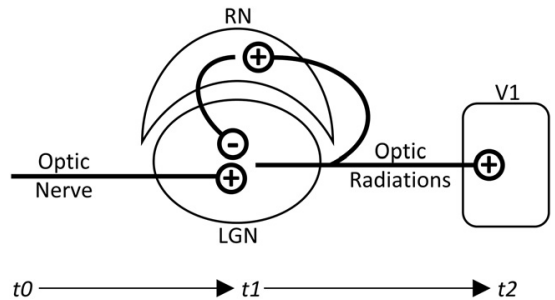

Figure 11. Visual pathway from retina to V1. The LGN/RN negative feedback loop provides a mechanism of cycling LGN excitability state. This LGN/RN network is known to give rise to sleep spindles. The same, or a similar, mechanism is expected to give rise the posterior $\alpha$ rhythm.

there is much evidence supporting this thalamic mechanism as the primary driver (Hughes and Crunelli, 2005). Although the present study does not directly test this thalamic mechanism, it is offered as a purely speculative explanation for our results on the basis of previous and more direct research findings (Lorincz et al., 2009; Chen et al., 2016).

\section{Cellular shutter effect generates a behavioral graded gating effect}

Although our speculation and not directly tested here, if the neural mechanism posited above were correct, the neuronic shutter effect would then best be described as occurring at the level of individual LGN relay cells, in which the effect may occur at a nearly binary level. However, the behavioral effects we observe are graded, with increasing and decreasing probabilities of stimulus observation. This may be explained given that oscillating excitatory states are not always synchronized across all LGN cells. It is not necessarily the case that all cells are simultaneously in burst-mode firing state; some may concurrently operate in the normal tonic firing state. Thus, one cell may be in a state of inhibition and not relay to $\mathrm{V} 1$, while others may relay visual information as usual. However, in asynchronous states, the $\alpha$ rhythm should be decreased in amplitude. As shown in this study, at higher $\alpha$ amplitudes, the differences in ORs between peak and trough phases at $t 2$ increased, presumably indicating that the measured phase becomes more representative of the excitatory state of larger populations of LGN cells with increasing $\alpha$ amplitude. Asynchrony is also important to consider, not just among cells within an LGN but between the LGNs of the left and right hemispheres. As on a cellular level, one LGN may restrict the flow of visual information while the other relays it. Presumably, if all cells within and between LGNs were in perfect synchrony, behavior relative to peak/trough phase at $t 2$ would reflect a perfect on/off visual shutter. This simple relationship was not observed in this study, and it is therefore more appropriate to describe the proposed neuronic shutter effect at the cellular level generating a graded, gating effect of perception at the behavioral level.

In sum, to our knowledge, this is the first study to examine the neuronic shutter effect with an exact phase and timing relationship to perceptual performance predicted by the underlying physiology of the visual system. The 
study design is novel and rigorous in controlling for individual visual conduction delays and hemispheric asynchrony. During times of high-amplitude $\alpha$ oscillations, we found participants on average are most likely to perceive stimuli at waveform trough $\left(272.41^{\circ}\right)$ with ORs $20.96 \%$ greater than at the opposing peak phase $\left(92.41^{\circ}\right)$. Given the rigor in our phase measurements, these results provide strong support for a mechanism that modulates perception described by the neuronic shutter effect and is reflected in the posterior $\alpha$ rhythm. Further studies with conduction delay-adjusted phase measurement are needed to investigate this shutter effect in the $\mu$ and tau rhythms over the sensorimotor and auditory cortices, to explore the possibility of this mechanism in other sensory systems.

\section{References}

Alexander GM, Carden WB, Mu J, Kurukulasuriya NC, McCool BA, Nordskog BK, Friedman DP, Daunais JB, Grant KA, Godwin DW (2006) The native T-type calcium current in relay neurons of the primate thalamus. Neuroscience 141:453-461.

American Electroencephalographic Society (1994) Guideline thirteen: guidelines for standard electrode position nomenclature. American Electroencephalographic Society. J Clin Neurophysiol 11:111-113.

Ancoli S, Green KF (1977) Authoritarianism, introspection, and alpha wave biofeedback training. Psychophysiology 14:40-44.

Barry RJ, Clarke AR, Johnstone SJ, Magee CA, Rushby JA (2007) EEG differences between eyes-closed and eyes-open resting conditions. Clin Neurophysiol 118:2765-2773.

Bazanova OM, Vernon D (2014) Interpreting EEG alpha activity. Neurosci Biobehav Rev 44:94-110.

Ben-Simon E, Podlipsky I, Okon-Singer H, Gruberger M, Cvetkovic D, Intrator N, Hendler T (2013) The dark side of the alpha rhythm: fMRI evidence for induced alpha modulation during complete darkness. Eur J Neurosci 37:795-803.

Berens P (2009) CircStat: a MATLAB toolbox for circular statistics. J Stat Soft 31:21.

Berry RB, Wagner MH (2015) Sleep medicine pearls, Ed 3. Philadelphia: Elsevier/Saunders.

Bierings RAJM, Kuiper M, Van Berkel CM, Overkempe T, Jansonius NM (2018) Foveal light and dark adaptation in patients with glaucoma and healthy subjects: a case-control study. PLoS One 13: e0193663.

Bland JM, Altman DG (1995) Multiple significance tests: the Bonferroni method. BMJ 310:170-170.

Bollimunta A, Chen Y, Schroeder CE, Ding M (2008) Neuronal mechanisms of cortical alpha oscillations in awake-behaving macaques. J Neurosci 28:9976-9988.

Bollimunta A, Mo J, Schroeder CE, Ding M (2011) Neuronal mechanisms and attentional modulation of corticothalamic $\alpha$ oscillations. J Neurosci 31:4935-4943.

Brainard DH (1997) The psychophysics toolbox. Spat Vis 10:433436.

Brüers S, VanRullen R (2018) Alpha power modulates perception independently of endogenous factors. Front Neurosci 12:279.

Callaway E, Alexander JD (1960) The temporal coding of sensory data: an investigation of two theories. J Gen Psychol 62:293-309.

Chen Z, Wimmer RD, Wilson MA, Halassa MM (2016) Thalamic circuit mechanisms link sensory processing in sleep and attention. Front Neural 9:83.

Clark VP, Fan S, Hillyard SA (1994) Identification of early visual evoked potential generators by retinotopic and topographic analyses. Hum Brain Mapp 2:170-187.

Cohen MX (2014) Analyzing neural time series data: theory and practice. Cambridge: MIT Press.

Cohen MX (2019) A better way to define and describe Morlet wavelets for time-frequency analysis. Neuroimage 199:81-86.
Connors BW, Amitai $Y$ (1997) Making waves in the neocortex. Neuron 18:347-349.

Cooper NR, Croft RJ, Dominey SJJ, Burgess AP, Gruzelier JH (2003) Paradox lost? Exploring the role of alpha oscillations during externally vs internally directed attention and the implications for idling and inhibition hypotheses. Int J Psychophysiol 47:65-74.

Cornsweet TN (1962) The staircase method in psychophysics. Am J Psychol 75:485-491.

Delorme A, Makeig S (2004) EEGLAB: an open source toolbox for analysis of single-trial EEG dynamics including independent component analysis. J Neurosci Methods 134:9-21.

Di Russo F, Martínez A, Sereno MI, Pitzalis S, Hillyard SA (2002) Cortical sources of the early components of the visual evoked potential. Hum Brain Mapp 15:95-111.

Dustman RE, Beck EC (1965) Phase of alpha brain waves, reaction time and visually evoked potentials. Electroencephalogr Clin Neurophysiol 18:433-440.

Ergenoglu T, Demiralp T, Bayraktaroglu Z, Ergen M, Beydagi $\mathrm{H}$, Uresin Y (2004) Alpha rhythm of the EEG modulates visual detection performance in humans. Cogn Brain Res 20:376-383.

Gonçalves SI, de Munck JC, Pouwels PJW, Schoonhoven R, Kuijer JPA, Maurits NM, Hoogduin JM, Van Someren EJW, Heethaar RM, Lopes da Silva FH (2006) Correlating the alpha rhythm to BOLD using simultaneous EEG/fMRI: inter-subject variability. Neuroimage 30:203-213.

Hughes SW, Crunelli V (2005) Thalamic mechanisms of EEG alpha rhythms and their pathological implications. Neuroscientist 11:357-372.

Hughes SW, Lörincz M, Cope DW, Blethyn KL, Kékesi KA, Parri HR, Juhász G, Crunelli V (2004) Synchronized oscillations at alpha and theta frequencies in the lateral geniculate nucleus. Neuron 42:253268.

lemi L, Busch NA, Laudini A, Haegens S, Samaha J, Villringer A, Nikulin VV (2019) Multiple mechanisms link prestimulus neural oscillations to sensory responses. Elife 8:e43620.

Janssens C, De Loof E, Nico Boehler C, Pourtois G, Verguts T (2018) Occipital alpha power reveals fast attentional inhibition of incongruent distractors. Psychophysiology 55:e13011.

Kizuk SA, Mathewson KE (2017) Power and phase of alpha oscillations reveal an interaction between spatial and temporal visual attention. J Cogn Neurosci 29:480-494.

Kleiner M, Brainard D, Pelli D, Ingling A, Murray R, Broussard C (2007) What's new in Psychtoolbox-3. Perception 36:1-16.

Klimesch W, Fellinger R, Freunberger R (2011) Alpha oscillations and early stages of visual encoding. Front Psychol 2:118.

Legewie H, Simonova O, Creutzfeldt OD (1969) EEG changes during performance of various tasks under open- and closed-eyed conditions. Electroencephalogr Clin Neurophysiol 27:470-479.

Lopes da Silva F (1991) Neural mechanisms underlying brain waves: from neural membranes. Electroencephalogr Clin Neurophysiol 79:81-93.

Lopez-Calderon J, Luck SJ (2014) ERPLAB: an open-source toolbox for the analysis of event-related potentials. Front Hum Neurosci 8:213.

Lorincz ML, Kékesi KA, Juhász G, Crunelli V, Hughes SW (2009) Temporal framing of thalamic relay-mode firing by phasic inhibition during the alpha rhythm. Neuron 63:683-696.

Mathewson KE, Gratton G, Fabiani M, Beck DM, Ro T (2009) To see or not to see: prestimulus alpha phase predicts visual awareness. J Neurosci 29:2725-2732.

Mathewson KE, Prudhomme C, Fabiani M, Beck DM, Lleras A, Gratton $G$ (2012) Making waves in the stream of consciousness: entraining oscillations in EEG alpha and fluctuations in visual awareness with rhythmic visual stimulation. J Cogn Neurosci 24:2321-2333.

Milton A, Pleydell-Pearce CW (2016) The phase of pre-stimulus alpha oscillations influences the visual perception of stimulus timing. Neuroimage 133:53-61. 
Omata K, Hanakawa T, Morimoto M, Honda M (2013) Spontaneous slow fluctuation of EEG alpha rhythm reflects activity in deep-brain structures: a simultaneous EEG-fMRI study. PLoS One 8:e66869.

Pelli DG (1997) The VideoToolbox software for visual psychophysics: transforming numbers into movies. Spat Vis 10:437-442.

Pfurtscheller G, Stancák A Jr, Neuper C (1996) Event-related synchronization (ERS) in the alpha band-an electrophysiological correlate of cortical idling: a review. Int J Psychophysiol 24:39-46.

Pineda JA (2005) The functional significance of mu rhythms: translating "seeing" and "hearing" into "doing." Brain Res Brain Res Rev 50:57-68.

Ray WJ, Cole HW (1985) EEG alpha activity reflects attentional demands, and beta activity reflects emotional and cognitive processes. Science 228:750-752.

Sauseng P, Klimesch W, Stadler W, Schabus M, Doppelmayr M, Hanslmayr S, Gruber WR, Birbaumer N (2005) A shift of visual spatial attention is selectively associated with human EEG alpha activity. Eur J Neurosci 22:2917-2926.

Seeck M, Koessler L, Bast T, Leijten F, Michel C, Baumgartner C, He B, Beniczky S (2017) The standardized EEG electrode array of the IFCN. Clin Neurophysiol 128:2070-2077.

Sherman SM (2001) Tonic and burst firing: dual modes of thalamocortical relay. Trends Neurosci 24:122-126.

Steriade M, Gloor P, Llinás RR, Lopes de Silva FH, Mesulam MM (1990) Basic mechanisms of cerebral rhythmic activities. Electroencephalogr Clin Neurophysiol 76:481-508.

Tenke CE, Kayser J, Svob C, Miller L, Alvarenga JE, Abraham K, Warner V, Wickramaratne P, Weissman MM, Bruder GE (2017) Association of posterior EEG alpha with prioritization of religion or spirituality: a replication and extension at 20-year follow-up. Biol Psychol 124:79-86.

Tiihonen J, Hari R, Kajola M, Karhu J, Ahlfors S, Tissari S (1991) Magnetoencephalographic $10-\mathrm{Hz}$ rhythm from the human auditory cortex. Neurosci Lett 129:303-305.

Timofeev I, Bazhenov M (2005) Mechanisms and biological role of thalamocortical oscillations. In: Trends in chronobiology research, pp 1-47. New York: Nova.

Timofeev I, Chauvette S (2011) Thalamocortical oscillations: local control of EEG slow waves. Curr Top Med Chem 11:2457-2471.

van Dijk H, Schoffelen JM, Oostenveld R, Jensen O (2008) Prestimulus oscillatory activity in the alpha band predicts visual discrimination ability. J Neurosci 28:1816-1823.

VanRullen R, Koch C (2003) Is perception discrete or continuous? Trends Cogn Sci 7:207-213.

VanRullen R, Zoefel B, Ilhan B (2014) On the cyclic nature of perception in vision versus audition. Philos Trans R Soc Lond B Biol Sci 369:20130214.

Weisz N, Hartmann T, Müller N, Lorenz I, Obleser J (2011) Alpha rhythms in audition: cognitive and clinical perspectives. Front Psychol 2:73-15.

Wieneke GH, Deinema CH, Spoelstra P, Storm van Leeuwen W, Versteeg H (1980) Normative spectral data on alpha rhythm in male adults. Electroencephalogr Clin Neurophysiol 49:636-645.

Yue W, Chun NW, Siong NK, Tiecheng W, Xiaoping L (2013) An EEG source localization and connectivity study on deception of autobiography memories. In: 2013 6th International IEEE/EMBS Conference on Neural Engineering (NER), pp 468-471. 